\title{
Erratum
}

\section{Effects of functional movement strength training on strength, muscle size, kinematics, and motor function in cerebral palsy: A 3-month follow-up}

Jung Ah Lee, Joshua H. You, Dong A. Kim, Min Jin Lee, Pil Woo Hwang, Nam Gi Lee, Jeong Joon Park, Dong Ryul Lee and Hyun-Kyung Kim

[NeuroRehabilitation 32 (2013), 287-295]

When this article was originally published, several of the affiliations were incorrectly given. The correct listing is as follows:

Jung Ah Lee ${ }^{\mathrm{a}}$, Joshua H. You ${ }^{\mathrm{b}}$, Dong A. Kimc, Min Jin Lee ${ }^{\mathrm{a}}$, Pil Woo Hwang ${ }^{\mathrm{a}}$, Nam Gi Lee ${ }^{\mathrm{d}}$, Jeong Joon Park ${ }^{\mathrm{c}}$, Dong Ryul Lee ${ }^{\mathrm{b}}$ and Hyun-Kyung Kim ${ }^{\mathrm{c}}$

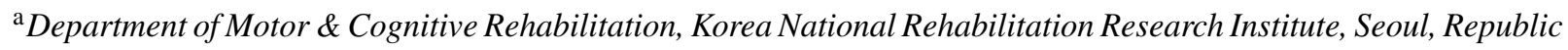
of Korea

${ }^{\mathrm{b}}$ Department of Physical Therapy, Yonsei University, Wonju City, Kangwon-do, Republic of Korea

${ }^{\mathrm{c}}$ Department of Rehabilitation Medicine, National Rehabilitation Hospital, Seoul, Republic of Korea

${ }^{\mathrm{d}}$ Department of Physical Therapy, College of Health and Welfare, Woosong University, Daejon, Republic of Korea 